Yo, Daniel Blake. Identidad, participación y política social

María Cristina Valencia Cepeda

Question/Cuestión, Nro.70, Vol.3, diciembre 2021

ISSN: 1669-6581

URL de la Revista: https://perio.unlp.edu.ar/ojs/index.php/question/

IICom -FPyCS -UNLP

DOI: https//doi.org/10.24215/16696581e613

\title{
Yo, Daniel Blake. Identidad, participación y política social
}

\section{I, Daniel Blake. Identity, participation and social policy.}

\section{María Cristina Valencia Cepeda}

Universidad Técnica de Ambato

Ecuador

cvalenciacepeda@yahoo.com.mx

https://orcid.org/0000-0003-2264-0976

\section{Resumen}

En este artículo se analiza cómo los procesos de políticas sociales construyen una verdad y en su ejecución definen subjetividades. A partir de la película: Yo, Daniel Blake, se define el silencio del Estado y de las políticas sociales frente a las demandas de los ciudadanos y su implicación en la subjetividad, aparecen las políticas sociales como dispositivos de constitución de los sujetos que los definen por lo que poseen y no por lo que son. Se considera como marco analítico el modelo de igualdad de oportunidades y el de igualdad de posiciones, definidos por Dubet, como dos lógicas diferentes para definir la identidad de los sujetos, pues mientras en el modelo de igualdad de oportunidades la identidad encadena, subordina y descalifica a los sujetos para conseguir asistencia social, en el modelo de igualdad de posiciones esa identidad representa una oportunidad de reciprocidad y participación. Se 
concluye que para que los procesos de participación social sean efectivos y justos se requiere el análisis crítico desde las políticas sociales sobre el lugar que se les asigna a los individuos beneficiarios de esas políticas, se requiere escuchar a esos individuos y construir una relación de reconocimiento de ese otro.

Palabras clave: Cine; Identidad: Política Social; Participación Social

\section{Abstract}

This article analyzes how social policy processes construct a truth and define subjectivities in their execution. From the film: I, Daniel Blake, the silence of the State and social policies is defined in the face of the demands of citizens and their involvement in subjectivity, then social policies appear as constitutional devices of the subjects that define them for what they possess and not for what they are. The model of equality of opportunities and that of equality of positions, defined by Dubet, are considered as an analytical framework as two different logics to define the identity of the subjects, because while in the model of equal opportunities identity chains, subordinates and disqualifies to the subjects to obtain social assistance, in the model of equality of positions that identity represents an opportunity for reciprocity and participation. It is concluded that for social participation processes to be effective and fair, critical analysis is required from social policies on the place assigned to the individuals who are beneficiaries of these policies, it is necessary to listen to these individuals and build a relationship of recognition of that other.

Keywords: Cinema; Identity; Social Policy; Social Participation

Cuando nos situamos en el mundo aparece el imperativo de definirnos, de nombrarnos, de responder ¿quién soy?, en este camino de búsqueda de certezas, aparecerán más dudas que certezas y nuestra identidad no hará más que enfrentarnos a las discontinuidades que nos atraviesan. Foucault dice que el individuo, es el producto de una relación de poder que se ejerce sobre los cuerpos, las multiplicidades, los movimientos, los deseos y las fuerzas (Foucault, 1979). Entonces nuestra identidad, aunque ilusoria, está inscrita en el juego de la construcción histórica del poder y la verdad, nuestra identidad es un efecto de esas verdades 
que los sistemas de poder producen y mantienen. En este análisis se plantea la manera en la que los procesos de políticas sociales construyen una verdad y en su ejecución definen subjetividades, sobre todo al no considerar las demandas de los ciudadanos, tomando como referencia la película Yo, Daniel Blake. Esa identidad entonces representa un limitante por un lado y por otro una posibilidad de participación social.

La identidad se define con y en el otro, cuando definimos ciertos elementos que nos unen con otros individuos, se forma un nosotros, que nos diferencia de ese otro, el nosotros de la ciudadanía, presenta posibilidades y limitaciones de participación social. La noción de ciudadanía que se apoya en la inscripción territorial, representa una noción en el plano jurídico, de derechos y de participación pero sobre todo en el plano identitario, que habilita a interactuar con el Estado como ciudadanos. Este plano subjetivo de la dimensión política de la territorialidad alude a un diverso espectro de fenómenos culturales y simbólicos, los cuales revisten de sentido a la institucionalización de las relaciones de poder y a los conflictos que dichas relaciones generan (Rofman, 2016). Sabemos, desde Marshall, que la ciudadanía tiene tres componentes: civil, político y social; el elemento civil consiste en los derechos necesarios para la libertad individual, como el derecho a la justicia desde la vía legal, el elemento político que refiere al ejercicio del poder político y el elemento social que tiene que ver con todo el espectro de bienestar y el derecho a participar socialmente (Marshall et al., 1997). Sin embargo, limitar la participación a la categoría de ciudadanía, aún de ciudadanía social, puede resultar excluyente, pues persiste la limitación de pertenencia territorial, lo que lleva a analizar el núcleo de la participación social cuyo objetivo es colectivizar tanto las prácticas de los sujetos sociales como las cuestiones relacionadas con las condiciones de vida y su producción (Clemente, 2016), entonces podemos entender la participación como una manera de hacer sociedad juntos, donde la singularidad de la identidad toma forma en la relación social con el otro (Rosanvallon, 2012).

En las políticas sociales ese otro es el Estado, la historia de Daniel Blake personifica la relación del individuo con el Estado, personifica esa voz de los ciudadanos frente a un Estado que no escucha. ¿Para qué escuchar a la gente si igual no lo escuchan a él?, dice Daysi cuando Dylan no le responde a Daniel Blake, añadiría a esta pregunta ¿para qué hablar si igual 
no escuchan?, ¿para qué hablar frente a un Estado sordo?. Cuando el personal de salud, que califica el subsidio por dispacidad que solicita Daniel, le hace preguntas que no guardan relación con el problema cardiaco y Daniel trata de indicarle a la operadora telefónica, pero ella no escucha. Cuando el funcionario indica que van a sancionar a Kattie por llegar tarde a la cita, aludiendo a sus obligaciones, Daniel les pregunta a los funcionarios, ¿por qué no la escuchan?, pero una vez más no hay respuesta a esta pregunta, una vez más no escuchan.

Entonces, tenemos por un lado la falta de respuesta, pero por otro lado, se evidencia esa respuesta a medias, a conveniencia desde la burocracia estatal, una respuesta que invita a esperar, a esperar en la incertidumbre. Auyero (2009) tomando como referencia a Bourdieu habla de la espera como parte integral del funcionamiento de la dominación. Cuando a Daniel Blake le llega la notificación de que no califica para el subsidio por discapacidad, decide llamar, la llamada, el tiempo, la espera, el juego, solo termina en incertidumbre, en más espera, no se sabe cuándo le llamaran dice la operadora de teléfono al terminar la conversación. Se asume entonces la espera como un mecanismo de producción y reproducción de la sumisión, es decir, que modifica de alguna manera el comportamiento de la persona que "aguanta", esa espera, que da cuenta de un proceso impregnado de incertidumbre, arbitrariedad que deviene en frustración, donde el Estado parece estar apuntando a la creación, de lo que Foucault denomina, un cuerpo dócil de beneficiarios (Auyero, 2009). China, el amigo de Daniel, le dice cuando busca apelar a la negativa del subsidio por discapacidad, "te harán la vida imposible", aludiendo de alguna manera a este proceso de espera, de incertidumbre y frustración que se desencadenará en el proceso.

El individuo no es una sustancia sino un devenir, Castel (2010) menciona que la consecuencia del nuevo régimen del capitalismo es el ascenso de las incertidumbres, las antiguas certezas identificadoras, como el trabajo se desvanecen, entonces, se define a estos individuos por lo que poseen y no por lo que son, los denominados individuos por exceso y por defecto. Se diría más bien que se define lo que son a partir de los medios con los que cuentan para enfrentar ese cambio, entonces hay individuos por exceso, que funcionan y salen a flote en el sistema, pero hay otros individuos que carecen de los soportes necesarios para poder afirmar un mínimo de independencia social, los individuos por defecto, que se someten a una 
dinámica de descalificación (Castel, 2010). Kattie y Daniel representan a esos individuos por defecto, cuya voz poco importa, individuos que frente a un modelo de Estado residual, de ayuda a los pobres basado en la comprobación de medios, se someten a "procesos sociales de deshonra", evidenciando el carácter performativo de las políticas en la identidad de los sujetos (Danani, 2017). Entonces, se reconoce a las políticas sociales como dispositivos de constitución de los sujetos (Rosanvallon, 2012). La ejecución de las políticas sociales, sobre todo, las denominadas, para "pobres" performan la identidad, se consideran los efectos de los denominados "acto de nombramiento", que se usan para clasificar, pues estas clasificaciones provocan efectos durables a nivel de las representaciones y permean las percepciones de los sujetos. De esta manera, los sujetos quedan encorsetados en las taxonomías y se definen por su condición de pobreza o vulnerabilidad, que les asigna una identidad, determina los relatos autobiográficos y las trayectorias de los sujetos "asistidos", ya sea para rechazar o resignificar estos atributos que han sido construidos para los sujetos asistidos (Soldano, 2009).

Una vez establecida la correlación entre las políticas sociales y la identidad, se consideran a continuación dos lógicas diferentes para definir la identidad de los sujetos, la del modelo de igualdad de oportunidades y la de igualdad de posiciones. El modelo de la igualdad de oportunidades considera la identidad asociada a la reivindicación de la discriminación e invita a demostrar que se es víctima de desventajas, construye un mundo de victimas donde cada individuo debe presentarse como si fuera más victima que los demás, porque de eso depende que sean más reconocidos y mejor asistidos. Aquí la identidad no representa libertad, pues me constituyo como víctima de mi identidad, porque esa identidad esta estigmatizada y se está obligado a someterse a ella, aunque fuera para combatir ese modo de discriminación (Dubet, 2011). Desde el modelo de oportunidades el contrato social se individualiza, les exhorta a ser un individuo, a hacerse cargo de sus trayectorias (Rosanvallon,2012), aunque no tengan los medios. Por tanto, se definen a las oportunidades como responsabilidades de los individuos, estos deben ser activos y movilizarse para merecer la ayuda, bajo esa lógica el subsidio se "trueca", como dice Dubet (2011), a cambio de responsabilidades y de compromisos. Daniel debe demostrar su compromiso, le indica la funcionaria para acceder al subsidio por desempleo, de manera, casi sagrada se alude al documento que firma con el Estado donde se compromete a buscar trabajo, puedo decir que no solo se compromete a 
buscar trabajo, sino a ser de la manera que el Estado le demanda, a ser un individuo activo, a demostrar que "merece" la ayuda. No se analiza entonces el error institucional de que Daniel Blake solicite subsidio por desempleo, cuando no puede trabajar, la responsabilidad recae en los individuos, a los que se les pide que quieran triunfar y aprovechar sus oportunidades, dejar de ser víctimas pasivas, incluso se les pide que asuman su fracaso (Dubet, 2011).

Además, este compromiso para recibir la prestación está atravesado por la lógica mercantil, es decir, nada de prestación sin contraprestación, lo que marca el triunfo del principio de individualización, que define que estas contraprestaciones deben aplicarse por igual a los individuos, aunque estén en situaciones diferentes, incluso las más difíciles (Rosanvallon, 2012). En la historia se aplica por "igual" la digitalización del sistema para pedir subsidio, aunque Daniel Blake acepta que nunca ha usado una computadora, pero todo el tiempo le repiten que todo se hace digital, no escuchan pero si imponen una nueva barrera, la tecnológica. Asimismo cuando Kattie llega tarde a la cita e indica que es porque no conoce la zona, se repite incansablemente que las reglas son para todos y no se considera su situación, o peor aún ni se escucha su argumento. Entonces, la lógica de la contraprestación demanda a ser, demanda a ser un tipo de individuo independientemente de la situación, diría que además la demanda no solo se trata de un compromiso, sino que se inscribe en la lógica del condicionamiento, pues la ayuda se da a cambio de la extinción de un castigo, en este caso, ser sancionado, donde se pone en evidencia la estructura de poder que se ejerce en esta dinámica desde la políticas sociales.

Cuando se le obliga a Daniel a asistir al taller de curriculum, se le invita a actuar para ser merecedor, se reproduce una vez más la noción de justicia social desde el modelo de oportunidades de Dubet (2011), considerando que este modelo implica no cuestionar el orden de las posiciones sociales, pero si cuestionar a los individuos que no alcanzan esas posiciones. La persona que da el taller de curriculum pone la siguiente situación: se requieren 8 empleados para una cafetería y se presentan más de 1300 currículos, ¿qué significa? pregunta el profesional, esperando un cuestionamiento que responsabilice a esos miles de personas que presentan su curriculum. Pero Daniel responde que es obvio que matemáticamente faltan trabajos, es decir, que vivimos en un mundo que pese a que pone al trabajo como eje central 
del bienestar, sabe que un porcentaje de la población no va a poder acceder a esos empleos. Sin embargo, en esta situación se insiste en la responsabilidad individual y no estructural, se produce entonces lo que describe Dubet (2011), cuando las posiciones se vuelven más escasas, cuando hay solo 8 vacantes para 1300 personas que necesitan trabajo, cuando la vida social empieza a parecerse a un juego de la silla, se discute menos sobre el número de las sillas que sobre las maneras de ocuparlas y sobre la equidad del árbitro (Dubet, 2011). En definitiva, si la responsabilidad es del individuo y no del sistema, el individuo debe pedir perdón o sentirse avergonzado, como Kattie en el banco de alimentos, aunque sea el sistema el que ha hecho que se muera de hambre, como Daniel a quien en el taller de curriculum se le insta a vivir en el "mundo real", a destacarse en el curriculum, pues no basta con mostrar capacidades se debe mostrar interés y dedicación, aunque el sistema no nos escuche.

En el modelo de igualdad de oportunidades se alude al concepto de autonomía, como la aceptación de que se posee algo y que se tiene la suficiente seguridad como para formular proyectos y sentirse libre (Dubet, 2011). Kattie quiere estudiar, asegura que no se rendirá, pero no depende de ella, ni Kattie ni Dan son libres, ni son reconocidos y tratados como individuos de pleno derecho (Rosanvallon, 2012). Kattie para comprarle unos zapatos a su hija Daysi empieza a prostituirse y Dan vende todas sus pertenencias para pagar su deuda, la lógica mercantil condiciona y atraviesa nuestras decisiones, incluso la lógica patriarcal, no hay autonomía, el cuerpo de la mujer como mercancía, ¿qué vender cuando no se tiene nada?. De tal forma que, no es que no tengan proyectos, pero les faltan los recursos para poder llevar a cabo sus proyectos y ser dueños de sus elecciones, como dice Rosanvallon dependen de la benevolencia de otro, de la asistencia pública que sin duda les concederá una ayuda, pero pagando un precio, que muchas de las veces es el aceptar que son incapaces de arreglárselas por sí mismos (Rosanvallon, 2012), aceptar que no tienen nada más. Daniel Blake dice: ¿cuándo ya no te respetas que te queda?, cuando la funcionaria le advierte sobre los riesgos de apelar al seguro de dispacidad, enfatizando que puede perderlo todo.

Entonces, ¿qué queda cuando no te escuchan, que queda frente a la espera, frente a la incertidumbre, , frente a una identidad menoscabada?. Es mi arte dice Daniel Blake, cuando escribe en la pared, cuando trata de que esas palabras en la pared le permitan ser escuchado, 
el arte como ese proceso de creación, como ese espacio de autonomía frente a un continuo cuestionamiento de la misma, como esa arma para ir en contra de la violencia estructural, como un ápice de dignidad. La noción de reconocimiento es fundamental para la participación social, porque es injusto que a algunos individuos y grupos se les niegue el status de plenos participantes en la interacción social, simplemente como consecuencia de su identidad y de manera específica de patrones institucionalizados de interpretación y valorización de las características distintivas que les asignan. Desde esta perspectiva, el reconocimiento es una cuestión de justicia, pues estos patrones de interpretación y de valoración impiden la paridad en la participación en la vida social (Fraser, 2008).

Por tanto, la identidad no solo puede representar un encadenamiento a esas categorías determinadas por la lógica del saber y el poder, según Dubet (2011) el modelo de igualdad de posiciones permite definir la identidad desde la libertad y no desde el encadenamiento. En el modelo de igualdad de posiciones elijo mi identidad por ella misma y no por su utilidad (Dubet, 2011). La identidad como libertad puede ser analizada desde la expectativa de una sociedad de iguales, donde la singularidad se afirma en relación con aquellas que le rodean (Rosanvallon, 2012), Daniel y Kattie, esos individuos exhortados por el Estado a hacerse cargo a pesar de no tener posibilidades en el mercado, ellos se encuentran, encuentran el uno en el otro una comunidad, un salvavidas siempre imperfecto, insuficiente frente al abandono del Estado y el mercado, pero que les permite construirse como sujetos, escucharse, que les permite mirarse, esa reciprocidad. En consecuencia, la identidad no adquiere vida sino en una democracia de reconocimiento de esa singularidad, en donde es posible la participación, como un común reflexivo que fortifica la vitalidad de una comunidad y del ser ciudadano, pues conduce a la igualdad (Rosanvallon, 2012).

En definitiva, la paridad participativa requiere dos condiciones, la primera tiene que ver con una condición objetiva de distribución material justa que asegure la independencia y la "voz" de los participantes y por otro lado, la condición intersubjetiva que tiene que ver con el cambio en los patrones culturales institucionalizados de interpretación y valoración que expresen igual respeto por todos los participantes y aseguren la igualdad. Por tanto, la propuesta de participación representa una norma universalista porque abarca a todos los 
participantes en la interacción, porque presupone el valor moral idéntico de los seres humanos, por esto es imposible la paridad participativa sin distribución justa y sin reconocimiento reciproco (Fraser, 2008).

En conclusión, se trata de separar el poder de la verdad de las formas de hegemonía sociales, económicas, y culturales en el interior de las cuales funciona (Foucault, 1979), se trata de dejar de lado la participación social de papel, donde las políticas son operaciones "técnicas" que reproducen formas de dominación nombrando y definiendo subjetividades subalternas, para así poder construir a ese otro de las políticas sociales desde el reconocimiento, como otro con un saber y una dignidad. Para esto es necesario aceptar que es posible que no sean a las personas a quienes tenemos que cambiar o asistir, aceptar que quizás para una verdadera participación tenemos que escuchar y que lo que se tenga que cambiar es, como dice Foucault, el régimen político, económico, institucional de la producción de la verdad. Cuando a Dylan le escuchan, él habla, escucha, juega con Dan, ya es escuchado entonces puede hablar, entonces puede escuchar al otro. Kattie en el funeral dice: Daniel para nosotros no era un pobre, no era un perro, era ese otro diferente al individuo de las políticas sociales, era ese individuo construido por esa reciprocidad de la mirada, de la palabra, de la escucha. Antes de la audiencia Daniel sigue preguntándose si le escucharan, si le escucharan esas personas para quienes no es más que un número de seguro, un nombre en una computadora, ellos que han definido su identidad, ¿le escucharan?. Nunca llega a escucharle, nunca serán conscientes de esa vida que estuvo en sus manos, y nosotros desde las ciencias sociales ¿seremos capaces de escuchar?.

\section{Referencias bibliográficas}

Auyero, J. (2009). Pacientes del estado. Un reporte etnográfico sobre la espera de la gente pobre. En Pacientes del Estado (pp. 228-228). Documento de Cátedra No 64.

Castel, R. (2010). El ascenso de las incertidumbres. Trabajo, protecciones, estatuto del.

Clemente, A. (2016). La participación como enfoque de intervención social. Participación, 
políticas públicas y territorio, 119.

Danani, C. (2017). Políticas sociales universales: Una buena idea sin sujeto Consideraciones sobre la pobreza y las políticas sociales. Revista Sociedad, 37.

Dubet, F. (2011). Repensar la justicia social: Contra el mito de la igualdad de oportunidades. Siglo Veintiuno Editores Argentina.

Foucault, M. (1979). Microfísica del poder, la Piqueta. Madrid.

Fraser, N. (2008). La justicia social en la era de la política de identidad: Redistribución, reconocimiento y participación. Revista de trabajo, 4(6).

Marshall, T. H., Casado, M. T., \& Miranda, F. J. N. (1997). Ciudadanía y clase social. Reis, 79, 297-344.

Rofman, A. (2016). Participación, políticas públicas y territorio: Aportes para la construcción de una perspectiva integral. Ediciones UNGS, Universidad Nacional de General Sarmiento.

Rosanvallon, P. (2012). La sociedad de iguales. Ediciones Manantial.

Soldano, D. (2009). El Estado en la vida cotidiana. Algunos desafíos conceptuales y metodológicos de la investigación sobre política y biografía. Frederic, S. y Soprano, G., Política y variaciones de escalas en el análisis de la Argentina. Buenos Aires: UNGS-Prometeo. 